\title{
Integrated modeling of protein-coding genes in the Manduca sexta genome using RNA-Seq data from the biochemical model insect
}

\author{
Xiaolong Cao, Haobo Jiang \\ ${ }^{a}$ Department of Entomology and Plant Pathology, Oklahoma State University, \\ Stillwater, OK 74078, USA
}

Key words: gene annotation; de novo assembly; tobacco hornworm; automated gene modeling; arthropod genomics.

Abbreviations: OGS, official gene set; ORF, open reading frame; L, length; ML, match length; QL, query length; SL, subject length; M, MAKER; C, Cufflinks; T, Trinity; O, Oases; U, UniProt Arthropoda; Y, C/T/O; S, similarity ratio of lengths; MLI, match length index; S1/S2, Selection 1 or 2; "P", perfect; "N", near perfect; "O", okay; "B", bad; "W", worst.

The sequence files of MCOT 1.0 transcripts and proteins are available to download at ftp://ftp.bioinformatics.ksu.edu/pub/Manduca/OGS2/OSU_files/. BLAST search of the two datasets can be performed at http://agripestbase.org/manduca/?q=blast.

Send correspondence to:

Haobo Jiang

Department of Entomology and Plant Pathology

Oklahoma State University

Stillwater, OK 74078

Telephone: (405)-744-9400

Fax: (405)-744-6039

E-mail: haobo.jiang@okstate.edu 


\section{Abstract}

The genome sequence of Manduca sexta was recently determined using 454 technology. Cufflinks and MAKER2 were used to establish gene models in the genome assembly based on the RNA-Seq data and other species' sequences. Aided by the extensive RNA-Seq data from 50 tissue samples at various life stages, annotators over the world (including the present authors) have manually confirmed and improved a small percentage of the models after spending months of effort. While such collaborative efforts are highly commendable, many of the predicted genes still have problems which may hamper future research on this insect species. As a biochemical model representing lepidopteran pests, $M$. sexta has been used extensively to study insect physiological processes for over five decades. In this work, we assembled Manduca datasets Cufflinks 3.0, Trinity 4.0, and Oases 4.0 to assist the manual annotation efforts and development of Official Gene Set (OGS) 2.0. To further improve annotation quality, we developed methods to evaluate gene models in the MAKER2, Cufflinks, Oases and Trinity assemblies and selected the best ones to constitute MCOT 1.0 after thorough crosschecking. MCOT 1.0 has 18,089 genes encoding 31,666 proteins: $32.8 \%$ match OGS 2.0 models perfectly or near perfectly, 11,747 differ considerably, and $29.5 \%$ are absent in OGS 2.0. Future automation of this process is anticipated to greatly reduce human efforts in generating comprehensive, reliable models of structural genes in other genome projects where extensive RNA-Seq data are available.

\section{Introduction}

With five larval instars, a large body size and hemolymph volume, and a simple larval body structure, the tobacco hornworm Manduca sexta has been widely employed as a model organism to study basic physiological processes in insects, such as cuticle formation, neural transmission, hormonal regulation, nutrient transport, intermediary metabolism, and immune responses 
(Hopkins et al., 2000; Shield and Hildebrand, 2001; Riddiford et al., 2003; Kanost et al., 1990; Arrese and Soulages, 2010; Jiang et al., 2010). Acquired knowledge of the molecular mechanisms underlying these processes would lead to new means of pest control, because $M$. sexta may be a good representative of some serious agricultural pests in the order of Lepidoptera. Several transcriptome analyses have yielded sequences and expression patterns of genes related to immunity, digestion, and olfaction (Zou et al., 2008; Pauchet et al., 2010; Zhang et al., 2011; Grosse-Wilde et al., 2011; Gunaratna and Jiang, 2013), but the potential of this model species is far from fulfillment partly due to the lack of its genome sequence. The shortage of complete protein sequences based on correctly modeled genes substantially hampers proteomic studies, for instance, of the immune complex formed around entomopathogens.

Recently, the genomic DNA isolated from a single male pupa of $M$. sexta was pyrosequenced at $>20$-fold coverage and assembled into Manduca Genome Assembly 1.0 (Msex 1.0) using Newbler with Atlas-GapFill (X et al., 2014). Sixty cDNA libraries, representing mRNA samples of whole larvae, organs and tissues at various developmental stages, were sequenced using Illumina technology, yielding >350 gigabyte data. Some of these RNA-Seq datasets and other known $M$. sexta cDNA sequences were aligned to the reference genome to generate Manduca Cufflinks Assembly 1.0 and 1.0b using Bowtie, TopHat, and Cufflinks. Aided by the available sequence data from M. sexta and other arthropod species, approximately 18,000 genes in Msex 1.0 were predicted by MAKER2 generating the Manduca Official Gene Set 1.0 (OGS 1.0). Some of the OGS 1.0 models were examined by annotators to detect errors using Manduca Cufflinks 1.0/1.0b, Trinity 3.0, and Oases 3.0 sequences. The latter two sets of gene transcripts, assembled solely based on the RNA-Seq datasets, were extensively used along with Cufflinks 1.0/1.0b to improve annotation quality. Over a period of more than one year, 2,498 structural genes were successfully curated by approximately 70 researchers (X et al., 2014). PASA2 (http://pasa. 
sourceforge.net/) was then used to select the best models from the MAKER2, Cufflinks, Trinity, Oases, and manual assemblies to generate Manduca OGS 2.0 (X et al., 2014).

During the course of gene cross-examination, we came to realize that some of the lessons learned can be valuable to future genome projects. For example, as extensive RNA-Seq data are becoming a norm, genome-dependent and independent assemblies are critically important in the validation and perfection of MAKER2 gene models. Due to limitations of the programs used to produce OGS 2.0 (Table 1), an integration of their outputs using computer programs may greatly reduce human efforts in sequence cross-examination and considerably increase the percentage of crosschecked gene models. To achieve these goals, we have developed methods to evaluate models in the MAKER, Cufflinks, Oases and Trinity assemblies. As proof of principle, a reliable, nearly complete set of protein sequences (MCOT 1.0) is generated to facilitate proteomic research in this model insect. In the following, we report the generation of Cufflinks 3.0, Oases 4.0 and Trinity 4.0 gene models, discuss their advantages, shortcomings and integration, and describe how MCOT 1.0 was developed and compared with OGS 2.0.

\section{Materials and Methods}

\subsection{Data and program acquisition}

Manduca Genome Assembly 1.0 (Msex 1.0) and gene models in Manduca Official Gene Sets 1.0 (OGS 1.0, Table S1) and 2.0 (OGS 2.0) and Cufflinks Assembly 1.0 (Cufflinks 1.0) (X et al., 2014) were downloaded from Manduca Base (ftp://ftp.bioinformatics.ksu.edu/pub/Manduca/). Universal protein sequences in UniProtKB Arthropoda (Table S1) were downloaded from ftp://ftp.ebi.ac.uk/pub/databases/fastafiles/uniprot/. The RNA-Seq datasets (X et al., 2014) were acquired from Dr. Gary Blissard at Cornell University. SAMtools (0.1.19) (Li et al., 2009), Bowtie2 (2.2.1) (Langmead and Salzberg, 2012), TopHat (2.0.11) (Trapnell et al., 2009), 
Cufflinks (2.1.1) (Trapnell et al., 2012; Roberts et al., 2011), Trinity (20131110) (Grabherr et al., 2011), Oases (0.2.08) (Schulz et al., 2012), and BLAST+ (2.2.29) (Camacho et al., 2009) were downloaded from $\quad$ http://samtools.sourceforge.net/, $\quad$ http://bowtiebio.sourceforge.net/bowtie2/index.shtml, $\quad$ http://ccb.jhu.edu/software/tophat/index.shtml, http://cufflinks.cbcb.umd.edu/, http://trinityrnaseq.sourceforge.net/, https://www.ebi.ac.uk/ zerbino/oases/, ftp://ftp.ncbi.nlm.nih.gov/blast/executables/blast+/LATEST/ and installed on a local supercomputer according to their manuals.

\subsection{Generation of Cufflinks 3.0}

The 60 RNA-Seq datasets were aligned to Msex 1.0 using TopHat at settings for three different read types: single end, paired end, and strand specific. "--read-realign-edit-dist 0" was selected to increase accuracy of read alignments. Cufflinks was used to translate the accepted hits generated by TopHat to separate GTF files, with the "-u" command enabled to allow more accurate handling of multiple reads mapped to the same region. Cuffmerge was employed to combine GTF files of all the libraries to make the final GFF file (see scripts in the Supplemental Materials), from which transcript sequences were extracted using gffread to form Cufflinks 3.0 dataset (Table S1).

\subsection{Reads treatment, normalization, and de novo assembling}

Paired end reads were trimmed to 80 bp using FASTX-Toolkit (http://hannonlab.cshl.edu/fastx_toolkit/index.html), with the forward reads combined in one file and the reverse ones in another. To handle the RNA-Seq data with 256 GB RAM of the supercomputer, the number of the reads was reduced according to Haas et al (2013). The Perl scripts provided in Trinity were used to perform in silico read normalization with maximum coverage set to 500. The single end and strand-specific reads were combined in one file for 
normalization at the same maximum coverage. After all normalized reads were pooled, Trinity was used to assemble the reads as paired end reads, generating Trinity 4.0 (Table S1). For Oases, four hash lengths $(k: 25,27,29,31)$ were chosen to assemble the reads as single end reads in four separate runs. Scaffolding was not allowed, preventing the stretches of Ns in assembled transcripts. The transcript files were then merged according to the Oases manual, generating Oases 4.0 (Table S1). In addition, reads that cannot be aligned to Msex 1.0 by TopHat were combined, trimmed to $80 \mathrm{bp}$, and assembled as paired end reads using Trinity. This new assembly (Trinity 4.0b, Table S1) was later used to identify unmapped genes, some of which may reside on the unsequenced $\mathrm{W}$ chromosome.

\subsection{Gene translation and sequence comparison}

Gene transcripts in Trinity 4.0 and Oases 4.0 were translated to polypeptide sequences using TransDecoder in Trinity (http://transdecoder.sourceforge.net/) (Haas et al., 2013), with minimum protein length set at 60 . For removing redundant sequences in the de novo assemblies, identical proteins were identified in one batch using Python scripts and only one of each group was kept in the final set of unique protein sequences. To identify the best sequences in the comparisons between assemblies, the BLOSUM62 scoring matrix in the BLAST source code was changed to -100 for all 190 non-identical residue pairs. As such, only identical or near identical sequences would be detected by BLASTP with a positive score of alignment. The gap opening penalty was set to the maximum $(32,767)$ to avoid gapped matches. A batchwise BLASTP comparison of the two sets of translated sequences was performed, with the tabular outputs (e.g. match length, query length, subject length) exported to Excel for further analysis. Cufflinks 3.0 translations were used as queries to search Trinity 4.0 or Oases 4.0 translations.

\subsection{Cross-examination and selection of protein sequences from different assemblies}

As illustrated in the flowchart (Fig. 1), the BLASTP results from comparisons of the unique 
protein sequences in Cufflinks 3.0, Trinity 4.0, and Oases 4.0 were examined by two methods to establish Selections 1 and 2. The results from one method were then cross-examined by the other to yield a dataset COT, later becoming a major part of MCOT 1.0.

In the length-based method, the Cufflink-Trinity comparison resulted in pairs with match lengths (TMLs, T for Trinity) and Cufflinks lengths (CLs), and their ratios were used to determine whether or not the Trinity hits would be kept (Fig. 1A). If TML/CL was $\leq 0.7$, the Trinity hits were ignored and corresponding Cufflinks sequences were further processed: for ones without ambiguous residues (Xs), their lengths (CLs) were directly used as CL*s; for the others with Xs, $70 \%$ of the CL values were used as CL*s. On the other hand, if TML/CL was > 0.7, the Trinity sequences were considered in the next step. The same procedure was carried out to compare Cufflinks and Oases translations and select the Oases ones (OML/CL > 0.7) for further consideration, together with the selected Cufflinks and Trinity sequences. The ones with the largest values (CL*, TL, or OL) were kept in Selection 1. If the values were equal, retention priority was given to the concerning sequences in the following order: Cufflinks, Trinity, and then Oases.

In the ratio-based method, Cufflinks 3.0 translations were used as queries to search arthropod universal/UniProt (U) sequences using BLASTP with the original BLOSUM62 matrix (Fig. 1B). Results were kept if identity $>35 \%$ and $\mathrm{ML} / \mathrm{QL}>0.7$ or $\mathrm{ML}>200$. When several regions were matched, ML equals the sum of match lengths between the same query and subject sequences. Up to five top hits were used to calculate UL (for UniProt length: mean \pm SD) and ID of the best match was kept. Lengths (CL, TL, OL and UL) of the Cufflinks 3.0, Trinity, Oases, and UniProt proteins, correlated by the BLASTP searches, were used to calculate similarity ratios CUS, TUS, and OUS. For example, TUS (i.e. Similarity ratio of lengths in a T-U comparison) was defined as TL/UL or UL/TL, whichever is between 0 and 1 , so that a TUS close to 1 indicates high 
similarity between this Trinity-UniProt pair. Depending on the absence or presence of Xs in the Cufflinks translations, CUS was directly used or adjusted to $70 \%$ as CUS*. The proteins with the highest ratios (CUS*, TUS or OUS) will be kept in Selection 2 and, if the values were equal, the priority order of $\mathrm{C}>\mathrm{T}>\mathrm{O}$ was used to determine which ones to retain.

To cross-examine the two selections, the length (L) and match length (ML) of sequence Y (C or T or $\mathrm{O}$ ) in Selection 2 (S2), UL of its correlated UniProt sequence, L and ML of its correlated sequence in Selection 1 (S1) were used to calculate $\mathrm{YUS}_{\mathrm{S} 2}-\mathrm{YUS}_{\mathrm{S} 1}$ (Fig. 1C). YUS $=\mathrm{L} / \mathrm{UL}$ or $\mathrm{UL} / \mathrm{L}$, whichever is 0 to 1 . Sequences in $\mathrm{S} 1$ were kept if their $\mathrm{YUS}_{\mathrm{S} 2}-\mathrm{YUS}_{\mathrm{S} 1}<0.3, \mathrm{ML}_{\mathrm{S} 1} / \mathrm{CL}>$ 0.95, or $\mathrm{ML}_{\mathrm{S} 1} / \mathrm{CL}>0.8$ when Cufflinks sequence contains Xs (route 1). Sequences in S2 were retained, if their $\mathrm{YUS}_{\mathrm{S} 2}-\mathrm{YUS}_{\mathrm{S} 1}>0.5$ and $\mathrm{L}_{\mathrm{S} 2} / \mathrm{CL}>0.7$ (route 2). The remaining sequence pairs most cases, $\mathrm{S} 1$ and $\mathrm{S} 2$ were identical $\left(\mathrm{YUS}_{\mathrm{S} 2}=\mathrm{YUS}_{\mathrm{S} 1}\right)$.

\subsection{Classification of sequence comparison results}

If the lengths of a query sequence (QL), subject sequence (SL), and match length (ML) were identical $(\mathrm{QL}=\mathrm{SL}=\mathrm{ML})$, the match was considered as "P" (for perfect). If $(\mathrm{ML} / \mathrm{QL}) \times(\mathrm{ML} / \mathrm{SL})>$ 0.95 (e.g. when ML $=$ QL, ML/SL > 0.95), the match was " $\mathrm{N}$ " (for near perfect). The $3^{\text {rd }}$ and $4^{\text {th }}$ categories "O" (for okay) and "B" (for bad) were separated based on match length index (MLI), defined as (ML/QL)/0.7 + ML/200. If MLI was $\geq 1$, the match was "O". In other words, even if of QL falls into the matched region is considerable. If MLI was $<1$, the match was "B". In the last category of "W" (for worst), the query sequences had no match. When OGS 1.0 and Cufflinks 3.0 datasets were compared, OGS 1.0 IDs with "B" and "W" matches were recorded.

\subsection{Identification of proteins present only in OGS 1.0}

Although accuracy of the gene models in OGS 1.0 is relatively low, some are unique (Table 
1). Since Cufflinks is more sensitive than Trinity and Oases (Yandell and Ence, 2012),

169

170

171

MAKER2 proteins were used as queries to search the Cufflinks 3.0 translations using BLASTP with the modified scoring matrix, according to Section 2.4. Based on the results, those sequences in the categories of "B" or "W" were stored as "M" (for MAKER2 unique proteins), later incorporated into MCOT 1.0.

\subsection{Identification of unmapped genes in Trinity $4.0 \mathrm{~b}$}

Since a male pupa was used for genome sequencing, genes located on the $\mathrm{W}$ chromosome are not present in Msex 1.0. In addition, the genome assembly probably lacks genes or gene pieces on other chromosomes, as gaps between scaffolds or NNN regions. Trinity $4.0 \mathrm{~b}$ was used to uncover transcripts of such unmapped genes. Based on results of the MCOT-Trinity 4.0b comparison, Trinity $4.0 \mathrm{~b}$ protein sequences in the categories of "B" or "W" were kept for BLASTP search of arthropod UniProt sequences using the original BLOSUM62 scoring matrix. Hits with ML > 100, identity > 35\%, and minimum/maximum of ML, QL and SL > 0.7 were combined with the proteins in "M" (Section 2.7) and "COT" (Section 2.5) to generate MCOT 1.0 (Table S1) after redundant sequences were removed. The redundant ones were identical sequences or shorter sequences (with zero or three residues trimmed off from both ends) identical to a part of longer ones.

\section{Results and discussion}

\subsection{Manduca Genome Assembly 1.0}

Shotgun sequencing of $M$. sexta genomic DNA fragments by the 454 technology resulted in a dataset at $>20$-fold of the genome size $(422 \pm 12 \mathrm{Mb})$, which was then assembled into Msex 1.0 (X et al., 2014). The genome assembly consists of 20,891 scaffolds (Table 2) with $\mathrm{N}_{50}$ at $664 \mathrm{~kb}$, much longer than the size of a typical lepidopteran insect gene. While this sequence set is good 
enough for gene modeling, other features may complicate the process: 1) $50.5 \%$ and $41.0 \%$ of the scaffolds are $<1 \mathrm{~kb}$ and $1 \mathrm{~kb}$ to $10 \mathrm{~kb}$, accounting for $1.70 \%$ and $4.05 \%$ of the $419 \mathrm{Mb}$ assembly size, respectively (Fig. 2A); 2) over 17,000 undetermined nucleotide (NNN) regions (average: 1,118 bp; range: 1-124,308 bp) (Fig. 2B) may contain genes or gene elements, even though they only account for $4.71 \%$ of the entire assembly; 3 ) conserved and novel repetitive elements, accounting for $25 \%$ of Msex 1.0 (X et al., 2014), and other highly similar sequences may cause errors in this assembly (Cao et al., 2014). Consequently, gene modeling can be a challenge in some cases.

\subsection{Manduca Cufflinks Assembly 3.0}

Cufflinks uses RNA-Seq data to model genes in a genome assembly (Table 1) (Trapnell et al., 2012; Roberts et al., 2011). We took advantage of Msex 1.0 and all 60 RNA-Seq datasets (X et al., 2014) to generate a new assembly, namely Cufflinks 3.0. As an update of Manduca Cufflinks 1.0, assembled using 33 of the 60 libraries, Cufflinks 3.0 contains 36,027 genes and 62,497 transcripts (Table 3). Cufflinks 1.0 has 37,281 genes and 64,301 transcripts. Perhaps, lacking RNA-Seq data support from scarcely expressed genes has split some genes and their transcripts into two or more pieces in Cufflinks 1.0. Analysis of Cufflinks 3.0 dataset indicates that $75 \%$ of the genes have one transcript form and $16 \%$ have 2 or 3 splicing alternates (Fig. 3). Thus, alternative splicing appears to be a minor concern for the genes predicted autonomously. In comparison, $96 \%$ of the MAKER2 gene models in OGS 1.0 have no splice variant, indicating this program is not good at predicting such variations.

\subsection{Trinity and Oases assemblies}

Based on the same reference genome, Cufflinks and MAKER2 may incorrectly predict genes if there are flaws in their corresponding genomic regions (Table 1, Section 3.1). To discover and repair this problem, we de novo assembled transcripts using the 60 RNA-Seq datasets. Totally, 

from 88,397 genes by Oases (Table 3). Due to characteristics of the Trinity and Oases programs (Table 1), the transcript numbers were 5.1 to 27.2-fold higher than those in Cufflinks 3.0 and OGS 1.0. The percentages of short transcripts $(<512 \mathrm{bp})$ were $48 \%$ in Trinity and $30 \%$ in Oases, much higher than $14 \%$ in Cufflinks 3.0 or OGS 1.0 (Fig. 4A, Table S2). Many of the short contigs in the genome-independent assemblies were probably caused by how these different programs handle problems such as single nucleotide polymorphisms, low quality reads, and posttranscriptional modifications. While Oases allows multiple hash levels, merging them does not necessarily produce a better assembly than Trinity did. The gene number was 88,397 or $45.8 \%$ of the Trinity models, but the protein number (total: 304,367 , unique: 130,474 ) was 1.95 - and 2.27-fold of the Trinity proteins (total: 155,825, unique: 57,593) (Table 3). Nonetheless, the numbers of transcripts and unique proteins in different size ranges (Fig. 4, A and B) did indicate that the RNA-Seq datasets were large and diverse enough for modeling a majority of the active genes and, in some cases, their splicing variants, all based on experimental evidence.

\subsection{Translation of the gene model sets}

We focus our efforts on structural genes to make $M$. sexta amenable to proteomic studies in the future. By translating their transcripts and setting the size limit to $>60$ residues, we expect to detect antimicrobial peptides (e.g. cecropins) but not some neuropeptides that are too small to tell apart from the noise of short open reading frames (ORFs). Some of the transcripts contain two or more ORFs, in most cases due to the merging of adjacent genes. As an extreme example, MAKER2 merged eleven adjacent genes into one coding for a gigantic "polyprotease". While the transcript numbers in Trinity and Oases are 5.1 and 8.8 times of that in Cufflinks, the numbers of unique proteins are just 1.5 to 3.5 times respectively (Table 3, Fig. 4B), suggesting that differences in the non-coding regions may also be responsible for the high transcript counts. 
Based on the protein size distribution (Table S3), Cufflinks outperforms the other three programs in modeling proteins longer than 2,049 residues, owing to its high sensitivity and reliance on Msex 1.0 (Table 1). The unique proteins shorter than 2,048 residues in Oases 4.0 are significantly higher in number than those in Trinity 4.0, then Cufflinks 3.0, and OGS 1.0 at last (Fig. 4B). Although part of this could be an artifact caused by Oases and Trinity to a lesser extent, the de novo assemblies well complement the other two assemblies by closing the gaps in Msex 1.0 (Table 1). MAKER2, primarily designed to model structural genes, has generated OGS 1.0. Albeit the smallest, this assembly contains unique genes. These genes are either scarcely expressed in the 52 tissue samples or expressed in unsampled tissues or stages so that they are not detected even by Cufflinks. In summary, an integration of the assemblies is necessary to generate a reliable, concise, and complete set of structural genes.

\subsection{Comparison of proteins in OGS 1.0 and Cufflinks 3.0}

To facilitate comparison among the four $M$. sexta assemblies, we modified the scoring matrix of BLASTP so that all non-identical residue pairs (e.g. Leu and Ile) score -100 (Section 2.4). Consequently, unless there is a long stretch of identical or near identical amino acid sequence in a query and a subject, the comparison always yields a negative score, allowing us to ignore the less-than-perfect matches that cause complications. After the proteins in OGS 1.0 and Cufflinks 3.0 were compared, 17,907 of the pairs were $100 \%$ identical, 226 were 98.0 to $99.9 \%$ identical, and these two groups together accounted for $99.95 \%$ of the total matches (Table 4). In this way, match length (ML) in the query (Q) and subject (S) were directly used to calculate (ML/QL) $\times(\mathrm{ML} / \mathrm{SL})$ and (ML/QL)/0.7 + ML/200 (i.e. MLI or match length index), without any concern about the exact percentage identity. The ML, QL, SL, (ML/QL) $\times(\mathrm{ML} / \mathrm{SL})$ and MLI values were then used to categorize the matches into "P", "N", "O", "B", and "W" (Section 2.6). Among the 22,310 unique proteins from the MAKER2 models, 6,481 perfectly and 2,245 near 
perfectly matched those from Cufflinks 3.0 (Table 5). Together, they account for $39.1 \%$ of the total. Another $39.1 \%$ fall into the "O" category. Proteins in the categories "B" (678) and "W" $(4,177)$ are considered to be unique, as they are not modeled by Cufflinks, Trinity, or Oases. The latter two are less sensitive than Cufflinks (Table 1).

\subsection{Comparison of proteins in Trinity 4.0, Oases 4.0, and Cufflinks 3.0}

Using the same method, we separately compared proteins in Cufflinks 3.0 with Trinity 4.0 and Oases 4.0 translations. Because translations of the MAKER2 models (Section 3.5), de novo assemblies, and arthropod UniProt sequences (Section 3.7) were all compared with translations of Cufflinks 3.0, identifications of the Cufflinks hits from these BLASTP searches serve as a liaison for all these datasets. The correlated protein sequences can then be evaluated to find the best model (Fig. 1).

In the comparison of Cufflinks 3.0 with Oases 4.0 translations, for example, $67.8 \%$ of the total matched sequences had ML/QL > 0.95 (Fig. 5A). The rest of hits fell into the realms of 0.95-0.7 (19.7\%) and 0.7-0 (12.5\%). We arbitrarily set the ML/QL threshold at 0.7 to identify Q and $\mathrm{S}$ sequences representing the same gene and kept the longer ones in Selection 1 (Fig. 1A). Likewise we found that $39.9 \%$ of the total O-C matches had (ML/QL) $\times(\mathrm{ML} / \mathrm{SL})>0.95$ (Fig. 5B); $1.7 \%$ of the total had (ML/QL)/0.7 + ML/200 (i.e. match length indices or MLIs) less than one (Fig. 5C). Using cutoff values of 1.0 for ML/QL, 0.95 for (ML/QL)×(ML/SL), and 1 for MLI, we categorized the matches into "P", "N", "O", "B" or "W". By correlating the results from T-C (Trinity 4.0 vs. Cufflinks 3.0) and O-C comparisons (Fig. 1A), we found 5,516 and 968 of the proteins in Cufflinks 3.0 perfectly and near perfectly matched both Trinity and Oases models (Table 6), respectively. Among the 37,316 total hits, 26,702 (71.6\%) fell into the same categories (P, N, O, B or W) from the comparisons, indicating that Trinity and Oases models are consistent in the protein-coding region at least. While 7,094 or $19.4 \%$ of the proteins were highly reliable 
(PP, NP, PN, and NN), 1,944 or $5.2 \%(\mathrm{BB}, \mathrm{BW}, \mathrm{WB}$, and $\mathrm{WW})$ were probably modeled by

Cufflinks only due to its high sensitivity (Table 1). The P/N/O proteins distributed normally over a broad size range; $68.7 \%$ of the $\mathrm{B} / \mathrm{W}$ were short ( $<128$ residues) (Table $\mathrm{S} 4$ and Fig. 6). Possibly

291

292

the short proteins came from untranslated regions of some genes, noncoding RNAs, or small protein genes expressed but undetected. In contrast to these extreme categories, 18,509 or $49.6 \%$ of the 37,316 total hits belong to the OO comparison and further efforts were made to select useful information from these sequences.

\subsection{Comparison of proteins in UniProtKB Arthropoda and Cufflinks 3.0}

Reliable proteins from other arthropods are useful for validating gene models. Therefore, we used BLASTP algorithm and the original BLOSUM62 matrix to compare query (Q) proteins in Cufflinks 3.0 translations with UniProtKB Arthropoda (i.e. UniProt or U) as described in Section 2.5. Of the 37,316 unique proteins in the Cufflinks 3.0, 30,313 or $81.2 \%$ had one to five matches; 7,003 had no match and may be unique in $M$. sexta. Their length distributions were normal distributions for the ones with 1 to 5 matches, but not so for those with 0 match (Table S5, Fig. 7) $-3,149$ or $45.0 \%$ of them were shorter than 128 residues. Some of the small proteins may not exist and it is also possible that BLASTP at the default settings has bias against short proteins. Nonetheless, assuming the sequence lengths of orthologous proteins are similar, we can exploit the links among UniProt, Cufflinks, Trinity, and Oases datasets to choose models by the ratiobased method to generate Selection 2 (Fig. 1B).

\subsection{Model selection among Cufflinks 3.0, Trinity 4.0, and Oases 4.0}

For all hits with ML/CL > 0.7, we chose the longest models for Selection 1 (S1, Fig. 1A, Section 2.5). When Xs (caused by NNNs) were present in the Cufflinks translations, the use of $\mathrm{CL}^{*}$ (i.e. 0.7CL), instead of $\mathrm{CL}$, allowed the de novo proteins to survive and replace the ambiguous Cufflinks models. To complement S1, lengths of the Trinity, Oases, Cufflinks, and 
UniProt $(\mathrm{U})$ proteins, correlated through Cufflinks IDs from the T-C, T-O, and T-U comparisons, were used to calculate the similarity ratios TUS, CUS* and OUS (Section 2.5, Fig. 1B). The models with ratios closest to 1.0 were kept in Selection 2 (S2). Cross-examination of the correlated proteins in $\mathrm{S} 1$ and $\mathrm{S} 2$ by ratio comparison $\left(\mathrm{YUS}_{\mathrm{S} 2}-\mathrm{YUS}_{\mathrm{S} 1}\right)$ resulted in the retention of 36,205 proteins without Xs (Fig. 1C, route 1). Crosschecking S2 contributed 35 proteins (route 2); manual checking improved the other 77 in S1 or S2 (route 3). Of the 999 sequences with Xs, 996 were selected via route 1 and three via route 2. Of 36,317 proteins without Xs, 29,612 have the same S1 and S2 result, and the rest, 6,593 keep S1 (route 1), 35 keep S2 (route 2) and only 77 needed manual checking (route 3).

\subsection{Generation of MCOT 1.0}

During the comparison of OGS 1.0 and Cufflinks 3.0 translations (Section 3.5), we found that $4,855 \mathrm{~B} / \mathrm{W}$ proteins in OGS 1.0 were not properly modeled by Cufflinks, possibly due to the limitation of detection sensitivity or scope. However, after these sequences were used as queries to search the de novo datasets with the length-based method, only 2,230 had $\mathrm{B} / \mathrm{W}$ matches in both Trinity 4.0 and Oases 4.0 translations; the other ones were P/N/O. Because some of the P/N/O proteins were detected in the Cufflinks transcripts by TBLASTN, we realized that, due to its settings, TransDecoder filtered out 2,625 proteins, accounting for $4.94 \%$ of the 53,102 Cufflinks 3.0 proteins. These 4,855 B/W proteins in OGS 1.0 were compared with translations of Trinity 4.0 and Oases 4.0 and model selection was performed as per the Cufflinks 3.0 translations.

After comparing Trinity 4.0 and Oases 4.0 translations with Cufflinks 3.0 translations (Section 3.8), we selected the best model for each of the 37,316 Cufflinks proteins (COT). Pooling the 4,855 MAKER2 models (M) with B/W matches to Cufflinks resulted in 42,171 IDs, some of which were selected more than once. After removing them, we found 35,567 IDs, 
removed 2,036 redundant sequences, eliminated 2,763 and 764 (100\% identical to a part of another after removal of 0 and 3 residues from each end, respectively), and obtained 30,004 protein sequences.

The intermediate BAM files generated by TopHat indicated that 20 to $30 \%$ of the RNA-Seq reads were not mapped to Msex 1.0 and may represent: 1) exons in the gaps, NNNs and W chromosome, 2) mitochondrial RNAs, or 3) others (e.g. polyA, mRNA of symbionts). To identify unmapped nuclear genes of $M$. sexta, we generated Trinity $4.0 \mathrm{~b}$ using the unmapped reads (Section 2.3) and adopted relatively strict standards to scrutinize the Trinity sequences (Section 2.8). Of the 39,809 unique proteins (> 60 residues) translated from Trinity 4.0b, 10,534 had no match $(\mathrm{W})$ with the 30,004 proteins; 212 had bad matches. In these 10,746 B/W proteins, only 1,183 (1,162 unique) had good UniProt matches. Some of the other 9,563 came from bacteria. The 1,162 proteins were combined with the 30,004 to generate MCOT 1.0. Of the 31,166 protein sequences in MCOT 1.0, 1,162 are from Trinity 4.0b, 7,118 are from Trinity 4.0, 2,559 from Oases 4.0, 3,715 from OGS 1.0 and 16,612 from Cufflinks 3.0. 31\% of those proteins from Trinity or Oases were updated from their original versions in Cufflinks 3.0 translations. 3.7\% were newly added genes in unsequenced genome regions including the $\mathrm{W}$ chromosome.

\subsection{Comparison of MCOT 1.0 with OGS 2.0}

There are 31,166 protein sequences in MCOT 1.0. Since they are originally from MAKER2, Cufflinks or Trinity $4.0 \mathrm{~b}$ models, we traced back to their gene IDs, and found 18,089 proteincoding genes gave rise to 28,449 transcripts after model selection and 31,166 proteins (Table 7). In comparison, there are 14,165 genes, 18,979 transcripts, and 20,888 proteins in OGS 2.0 (after being filtered by the same method for MCOT 1.0). There are $21.7 \%$ fewer genes, $29.7 \%$ fewer transcripts and $33.0 \%$ fewer proteins in OGS 2.0 compared to MCOT 1.0 after counting genes, transcripts and proteins with exactly the same standard as MCOT 1.0. We then used the protein 
sequences in MCOT 1.0 as queries to search OGS 2.0 using BLASTP and the modified scoring matrix. The results showed 8,034 P, 2,178 N, 11,747 O, $996 \mathrm{~B}$, and 8,211 W, indicating that $32.8 \%$ were $\mathrm{P} / \mathrm{N}, 37.7 \%$ were $\mathrm{O}$, and $29.5 \%$ were $\mathrm{B} / \mathrm{W}$ (Table 8 ). The differences are substantial between the two assemblies. MCOT 1.0 is more inclusive than OGS 2.0 in terms of covering proteins. To facilitate the usage of MCOT 1.0 for proteomic studies, we have developed a naming system, which provides information of their sources, identification, matching qualities, and reference to OGS 2.0 (Fig. 8).

\subsection{Additional information from Cufflinks 3.0}

A major part of MCOT 1.0 is refined from Cufflinks 3.0 models which includes 36,027 genes and 62,497 transcripts (Table 3). Using Transdecoder, we found 20,289 of the Cufflinks genes were not translated to proteins (based on the definition in Section 2.4), suggesting that most of them are noncoding. While 22,615 of the Cufflinks genes are absent in MCOT 1.0, the difference of 2,326 indicated that some of them may have been correctly merged during MCOT 1.0 generation. Of the 20,289 noncoding genes, the most complex gene $(4,000 \mathrm{bp}$ in length, $71.5 \%$ of $\mathrm{A} / \mathrm{T}$ ) have 33 alternative splicing forms and could be a long, noncoding RNA. Length distributions of the coding and noncoding transcripts in Cufflinks 3.0 (Fig. 9) were strikingly different: the coding ones are a lot longer. Surprisingly, 4,144 noncoding genes are 2,049 to 8,192 bp and 183 are > 8,193 bp. While MCOT 1.0 focuses on structural genes, the non-coding genes are another world to explore in the future.

\subsection{Summary}

We developed an integrated approach to select the best models based on BLASTP comparison of the Cufflinks dataset with sequences in OGS 1.0 and the de novo assemblies. The modified scoring matrix greatly simplified the sequence comparison by keeping pairs with $>98 \%$ identity. Correlated by Cufflinks IDs, the models in different assembles (Trinity 4.0, Oases 4.0, 
OGS 1.0, and UniProt) were compared and chosen based on length-derived parameters. By incorporating unique sequences in OGS 1.0 and unmapped genes in the Trinity $4.0 \mathrm{~b}$, we generated MCOT 1.0, which has $60 \%$ more proteins than OGS 2.0. As extensive RNA-Seq data are available for most genome projects nowadays, automation of our procedures will produce comprehensive models of protein-coding genes in the future.

\section{Acknowledgements}

This study was supported by NIH grant GM58634. We thank Drs. Ulrich Melcher and Jamie Walters for their critical comments of the manuscript. The Manduca Genome Project, which provided Msex 1.0, OGS 1.0, OGS 2.0, Cufflinks 1.0, and RNA-Seq datasets, was funded by DARPA (Gary Blissard, Boyce Thompson Institute) and NIH grant GM41247 (Michael Kanost, Kansas State University). This work was approved for publication by the Director of Oklahoma Agricultural Experimental Station, and supported in part under project OKLO2450 (to H. Jiang). Computation for this project was performed at OSU High Performance Computing Center supported in part through NSF grant OCI-1126330.

\section{References}

Arrese, E.L., Soulages, J.L., 2010. Insect fat body: energy, metabolism, and regulation. Ann Rev Entomol. 55, 207-225.

Camacho, C., Coulouris, G., Avagyan, V., Ma, N., 2009. BLAST+: architecture and applications. BMC Bioinformatics. 10, 421.

Cao, X., He, Y., Hu, Y., Zhang, X., Wang, Y., Zou, Z., Chen, Y., Blissard, G.W., Kanost, M.R., Jiang, H., 2014. Sequence conservation, phylogenetic relationships, and expression profiles of nondigestive serine proteases and serine protease homologs in Manduca sexta. in review

Grabherr, M.G., Haas, B.J., Yassour, M., Levin, J.Z., Thompson, D.A., Amit, I., Adiconis, X., Fan, L., Raychowdhury, R., Zeng, Q., Chen, Z., Mauceli, E., Hacohen, N., Gnirke, A., Rhind, 
N., Palma, F. di, Birren, B.W., Nusbaum, C., Lindblad-Toh, K., Friedman, N., Regev, A., 2011. Full-length transcriptome assembly from RNA-Seq data without a reference genome. Nat Biotechnol. 29, 644-652.

Grosse-Wilde, E., Kuebler, L.S., Bucks, S., Vogel, H., Wicher, D., Hansson, B.S., 2011. Antennal transcriptome of Manduca sexta. Proc Natl Acad Sci USA. 108, 7449-7454.

Gunaratna, R. Jiang, H., 2013. A comprehensive analysis of Manduca sexta immunotranscriptome. Dev Com Immunol. 39, 388-398.

Haas, B.J., Papanicolaou, A., Yassour, M., Grabherr, M., Blood, P.D., Bowden, J., Couger, M.B., Eccles, D., Li, B., Lieber, M., Macmanes, M.D., Ott, M., Orvis, J., Pochet, N., Strozzi, F., Weeks, N., Westerman, R., William, T., Dewey, C.N., Henschel, R., Leduc, R.D., Friedman, N., Regev, A., 2013. De novo transcript sequence reconstruction from RNA-seq using the Trinity platform for reference generation and analysis. Nat Protoc. 8, 1494-1512.

Hopkins, T., Krchma, L., Ahmad, S., Kramer, K., 2000. Pupal cuticle proteins of Manduca sexta: characterization and profiles during sclerotization. Insect Biochem Mol Biol. 30, 19-27.

Jiang, H., Vilcinskas, A., Kanost, M.R., 2010. Immunity in lepidopteran insects. In "Invertebrate Immunity” (K. Söderhäll ed.), Adv Exp Med Biol. 708, 181-204.

Kanost, M.R., Kawooya, J.K., Law, J.H., Ryan, R.O., Van Heusden, M.C., Ziegler, R., 1990. Insect hemolymph proteins. Adv Insect Physiol. 22, 299-396.

Langmead, B, Salzberg, SL, 2012. Fast gapped-read alignment with Bowtie 2. Nat Methods. 9, 357-359.

Li, H., Handsaker, B., Wysoker, A., Fennell, T., Ruan, J., Homer, N., Marth, G., Abecasis, G., Durbin, R., 2009. The Sequence Alignment/Map format and SAMtools. Bioinformatics. 25, 2078-2079.

Pauchet, Y., Wilkinson, P., Vogel, H., Nelson, D.R., Reynolds, S.E., Heckel, D.G., ffrenchConstant, R.H., 2010. Pyrosequencing the Manduca sexta larval midgut transcriptome: messages for digestion, detoxification and defence. Insect Mol Biol. 19, 61-75.

Riddiford, L., Hiruma, K., Zhou, X., Nelson, C.A., 2003. Insights into the molecular basis of the hormonal control of molting and metamorphosis from Manduca sexta and Drosophila melanogaster. Insect Biochem Mol Biol. 33, 1327-1338.

Roberts, A., Trapnell, C., Donaghey, J., Rinn, J.L., Pachter, L., 2011. Improving RNA-Seq expression estimates by correcting for fragment bias. Genome Biol. 12, R22. 
Schulz, M.H., Zerbino, D.R., Vingron, M., Birney, E., 2012. Oases: robust de novo RNA-seq assembly across the dynamic range of expression levels. Bioinformatics. 28, 1086-1092.

Shields, V., Hildebrand, J.G., 2001. Recent advances in insect olfaction, specifically regarding the morphology and sensory physiology of antennal sensilla of the female sphinx moth Manduca sexta. Microsc Res Tech. 55, 307-329.

Trapnell, C, Pachter, L, Salzberg, SL, 2009. TopHat: discovering splice junctions with RNA-Seq. Bioinformatics. 25, 1105-1111.

Trapnell, C., Roberts, A., Goff, L., Pertea, G., Kim, D., Kelley, D.R., Pimentel, H., Salzberg, S.L., Rinn, J.L., Pachter, L., 2012. Differential gene and transcript expression analysis of RNA-seq experiments with TopHat and Cufflinks. Nat Protoc. 7, 562-578.

X et al., 2014.

Yandell, M., Ence, D., 2012. A beginner's guide to eukaryotic genome annotation. Nat Rev Genet. 13, 329-342.

Zhang, S., Gunaratna, R.T., Zhang, X., Najar, F., Wang, Y., Roe, B., Jiang, H., 2011. Pyrosequencing-based expression profiling and identification of differentially regulated genes from Manduca sexta, a lepidopteran model insect. Insect Biochem Mol Biol. 41, 733 746.

Zou, Z., Najar, F., Wang, Y., Roe, B., Jiang, H., 2008. Pyrosequence analysis of expressed sequence tags for Manduca sexta hemolymph proteins involved in immune responses. Insect Biochem Mol Biol. 38, 677-682.

Table 1. Comparison of the four gene prediction programs

\begin{tabular}{|c|c|c|c|}
\hline Program & Algorithm & Advantages & Disadvantages \\
\hline Cufflinks & $\begin{array}{l}\text { map reads to the reference genome } \\
\text { with TopHat and Bowtie to identify } \\
\text { splice sites, and then use outputs of } \\
\text { TopHat to create gene models }\end{array}$ & $\begin{array}{l}\text { most sensitive; accurate splicing } \\
\text { sites; GTF file for gene annotation; } \\
\text { fast, less computation; more tolerant } \\
\text { to low quality reads }\end{array}$ & $\begin{array}{l}\text { carry errors in the genome scaffolds } \\
\text { (gaps, NNNs, misassembling, etc.); } \\
\text { many isoforms from closely located } \\
\text { and related genes do not exist }\end{array}$ \\
\hline Maker2 & $\begin{array}{l}\text { align EST and protein sequences to } \\
\text { genome to produce ab initio gene } \\
\text { predictions and can use RNA-Seq } \\
\text { data to improve the prediction. }\end{array}$ & $\begin{array}{l}\text { less redundant; model genes poorly } \\
\text { represented in the RNA-Seq } \\
\text { datasets; GTF file for gene } \\
\text { annotation }\end{array}$ & $\begin{array}{l}\text { low quality of predictions, such as } \\
\text { extra or skipped exons, inaccurate } \\
\text { splicing junctions, and merging of } \\
\text { adjacent genes; biased on proteins }\end{array}$ \\
\hline Trinity & $\begin{array}{l}\text { De novo assemble transcripts using } \\
\text { RNA-Seq data }\end{array}$ & $\begin{array}{l}\text { not influenced by problems in the } \\
\text { genome assembly }\end{array}$ & $\begin{array}{l}\text { single hash level ( } k \text { : } 25) \text {; less } \\
\text { sensitive than Cufflinks; redundant } \\
\text { transcripts; no GTF file; SNPs etc. }\end{array}$ \\
\hline Oases & $\begin{array}{l}\text { De novo assemble transcripts using } \\
\text { RNA-Seq data, and use Velvet for } \\
\text { contig assembling }\end{array}$ & $\begin{array}{l}\text { accurate, not influenced by } \\
\text { problems in the genome assembly, } \\
\text { multiple hash levels to improve }\end{array}$ & $\begin{array}{l}\text { less sensitive than Cufflinks, } \\
\text { redundant transcripts; intense } \\
\text { computation for large datasets; no }\end{array}$ \\
\hline
\end{tabular}


Table 2. Summary statistics of $M$. sexta scaffolds in Msex 1.0 (data from X et al., 2014)

\begin{tabular}{cccccccc}
\hline size range & number & $\begin{array}{c}\text { \% of total } \\
\text { number }\end{array}$ & length & $\begin{array}{c}\% \text { of total } \\
\text { length }\end{array}$ & $\begin{array}{c}\text { NNN } \\
\text { number }\end{array}$ & NNN length & $\begin{array}{c}\% \text { of NNN } \\
\text { length }\end{array}$ \\
\hline$<1 \times 10^{3}$ & 10,543 & 50.5 & $7,516,906$ & 1.8 & 13 & 13 & 0.00 \\
$10^{3}-10^{4}$ & 8,572 & 41.0 & $16,986,901$ & 4.1 & 340 & 551,049 & 3.24 \\
$10^{4}-10^{5}$ & 1,083 & 5.2 & $40,475,711$ & 9.6 & 3,568 & $4,970,857$ & 12.28 \\
$10^{5}-10^{6}$ & 604 & 2.9 & $209,932,343$ & 50.0 & 9,576 & $10,185,979$ & 4.85 \\
$>10^{6}$ & 89 & 0.4 & $144,530,018$ & 34.5 & 4,188 & $4,061,178$ & 2.80 \\
\hline total & 20,891 & 100 & $419,441,879$ & 100 & 17,685 & $19,769,076$ & 4.71 \\
\hline
\end{tabular}

Table 3. Numbers of genes, transcripts, and proteins predicted by different programs

\begin{tabular}{cccccc}
\hline program & assembly & genes & transcripts & proteins & unique proteins \\
\hline MAKER2 & OGS 1.0 & 18,750 & 20,317 & 22,310 & 22,310 \\
Cufflinks & Cufflinks 3.0 & 36,027 & 62,497 & 53,102 & 37,316 \\
Trinity & Trinity 4.0 & 193,161 & 317,062 & 155,825 & 57,593 \\
Oases & Oases 4.0 & 88,397 & 552,733 & 304,367 & 130,474 \\
\hline
\end{tabular}

Table 4. Distribution of numbers of matched proteins over sequence identity in the BLASTP comparison of the protein sequences in OGS 1.0 and Cufflinks 3.0

\begin{tabular}{ccc}
\hline Identity $(\%)$ & Count & $\%$ of total counts \\
\hline $96-97$ & 1 & 0.01 \\
$97-98$ & 8 & 0.04 \\
$98-99$ & 58 & 0.32 \\
$99-100$ & 168 & 0.94 \\
100 & 17,672 & 98.69 \\
\hline
\end{tabular}

Table 5. BLASTP comparison of OGS 1.0 and Cufflinks 3.0 models

\begin{tabular}{ccc}
\hline category & count & \% of total counts \\
\hline $\mathrm{P}$ (perfect) & 6,481 & 29.05 \\
$\mathrm{~N}$ (near perfect) & 2,245 & 10.06 \\
$\mathrm{O}$ (okay) & 8,729 & 39.13 \\
$\mathrm{~B}$ (bad) & 678 & 3.04 \\
$\mathrm{~W}$ (worst) & 4,177 & 18.72 \\
\hline total & 22,310 & 100 \\
\hline
\end{tabular}

Table 6. BLASTP comparison of Cufflinks 3.0, Trinity 4.0, and Oases 4.0 models

\begin{tabular}{|c|c|c|c|c|c|c|}
\hline \multirow{2}{*}{\multicolumn{2}{|c|}{ Cufflinks }} & \multicolumn{5}{|c|}{ Oases } \\
\hline & & $P$ & $\mathrm{~N}$ & 0 & $B$ & W \\
\hline \multirow{4}{*}{ Trinity } & $P$ & 5,516 & 407 & 3,490 & 178 & 228 \\
\hline & $\mathrm{N}$ & 203 & 968 & 1,511 & 39 & 22 \\
\hline & $\mathrm{O}$ & 1,592 & 824 & 18,509 & 796 & 361 \\
\hline & B & 22 & 6 & 213 & 325 & 89 \\
\hline
\end{tabular}




\begin{tabular}{l|l|lllll}
\hline W & 151 & 21 & 315 & 146 & 1,384 \\
\hline
\end{tabular}

Table 7. Summary statistics of MCOT 1.0 and OGS 2.0

\begin{tabular}{c|cc}
\hline & MCOT 1.0 & OGS 2.0 \\
\hline gene \# & 18,089 & 14,165 \\
transcript \# & 28,449 & 18,979 \\
final protein \# & 31,166 & 20,888 \\
\hline
\end{tabular}

Table 8. Comparison of MCOT 1.0 and OGS 2.0

\begin{tabular}{c|ccccc}
\hline Query to Subject & P & N & O & B & W \\
\hline MCOT1.0 to OGS2.0 & 8,034 & 2,178 & 11,747 & 996 & 8,211 \\
\hline
\end{tabular}

\section{Figure legends}

Fig. 1. Scheme of sequence comparison and selection. A) The length-based comparison of Cufflinks (C), Trinity (T), and Oases (O) protein sequences to generate Selection $1(\mathrm{~S} 1)$. B) The ratio-based comparisons (C-U, T-U and O-U) to generate Selection 2 (S2). C) Crossexamination of S1 and S2 to generate COT, a major component of MCOT 1.0.

Fig. 2. Length distributions of Scaffolds and NNN regions. A) Percentage of scaffold numbers and sizes; B) lengths of NNN regions and corresponding scaffolds.

Fig. 3. Percentages of genes with $1,2,3,4,5$, or $\geq 6$ splicing forms based on Cufflinks 3.0 (left) and MAKER2-generated OGS 1.0 (right)

Fig. 4. Size distributions of transcripts (A) and unique proteins (B) predicted by the four programs.

Fig. 5. Distributions of ML/QL (A), (ML/QL) $\times(\mathrm{ML} / \mathrm{SL})(\mathbf{B})$, and (ML/QL)/0.7 + ML/200 (C) values from Cufflinks-Oases comparison.

Fig. 6. Size distributions of unique Cufflinks proteins in the $P / N / O$ (red) and $B / W$ (grey) categories after comparison with the de novo assemblies.

Fig. 7. Size distributions of unique Cufflinks proteins with $0,1,2,3$, 4, and $\geq 5$ UniProt hits.

Fig. 8. Naming of MCOT 1.0 sequences. In gene "MCOT.X\#", $X$ stands for "M" (MAKER2), "C" (Cufflinks 3.0) or "W" (Trinity 4.0b) to indicate its original source (before BLAST search and model selection), and \# is the 5-digit ID (e.g. 02367). Transcripts are named "MCOT.X\#.\#", where the second \# $(1,2 \ldots)$ stands for the $1^{\text {st }} / 2^{\text {nd }} / \ldots$ transcript from the same gene. Likewise 
proteins are named "MCOT.X\#.\#.\#.XYZ\#V", where the third \# represents the $1^{\text {st }} / 2^{\text {nd }} / \ldots$ protein

420 from the same transcript. If one gene generates one transcript and then one protein, the second 421 and third \#'s are marked as " 0 ”. Multicistronic genes are rare, but do exist in insects. The $2^{\text {nd }} \mathrm{X}$ 422 indicates the final sequence source of "M", "C", "T (Trinity 4.0)", "O" (Oases 4.0), or "W" 423 (unmapped, including those on the $\mathrm{W}$ chromosome). $\mathrm{Y}$ and $\mathrm{Z}$ are the quality of matching with 424 Trinity 4.0 and Oases 4.0, respectively: "P" (perfect), "N" (near perfect), "O” (okay), "B” (bad), 425 "W" (worst), or "X" (data unavailable). The fourth \# is the number of kept UniProt hits (0 to 5 or $426 \mathrm{X}$ for data unavailable). V marks the quality of matching with OGS 2.0: if "P", "N" or "O", the 427 corresponding OGS 2.0 ID is added next to "l"; otherwise " $\mathrm{X}$ " is added to indicate no good 428 match.

429 Fig. 9. Size distributions of the coding and noncoding transcripts in Cufflinks 3.0.

\section{Supplemental materials}

Scripts for combining GTF files of all the libraries:

tophat -p 12 --read-realign-edit-dist 0 -o StrandSpecific --library-type fr-firststrand Msgenome StrandSpecific.fastq tophat -p 12 --read-realign-edit-dist 0 -o SingleEnd Msgenome SingleEnd.fastq tophat - $p 12$--read-realign-edit-dist 0 -o PairedEnd Msgenome PairedEnd-f.fastaq PairedEnd-r.fastaq cufflinks -p 12 -b Msgenome.fa -u -o output accepted_hits.bam cuffmerge -s Msgenome.fa -p 12 -o merge gtf_list.txt

Table S1. Datasets generated or used in this study and their descriptions

\begin{tabular}{c|l}
\hline Name of Dataset & \multicolumn{1}{c}{ Description } \\
\hline Msex 1.0 & Manduca Genome Assembly 1.0 generated by Newbler with Atlas-GapFill \\
Cufflinks 3.0 & RNA-Seq reads aligned to the genome by TopHat; genes modeled by Cufflinks \\
Trinity 4.0 & RNA-Seq reads normalized and assembled by Trinity \\
Trinity 4.0b & Trinity assembly of RNA-Seq reads not aligned to the genome \\
Oases 4.0 & RNA-Seq reads normalized and then assembled by Oases with four different hash lengths \\
OGS 1.0 & Genes modeled based on the genome sequence by MAKER2 \\
OGS 2.0 & OGS 1.0 improved by manual annotation and PASA2 using Cufflinks and de novo assemblies \\
Uniprot Arthropoda & Downloaded from Uniprot database on April 15, 2014 \\
MCOT 1.0 & Assembled using Msex 1.0, Cufflinks 3.0, Trinity 4.0 \& 4.0b, Oases 4.0, and Uniprot Arthropoda \\
\hline
\end{tabular}

Table S2. Transcript length distribution of different modeling programs 


\begin{tabular}{c|cccc|cccc}
\hline \multirow{2}{*}{$\begin{array}{c}\text { size range } \\
\text { (bp) }\end{array}$} & \multicolumn{4}{|c|}{ frequency } & \multicolumn{4}{c}{ percentage } \\
\cline { 2 - 9 } & MAKER2 & Cufflinks & Trinity & Oases & MAKER2 & Cufflinks & Trinity & Oases \\
\hline$<128$ & 64 & 1,391 & 0 & 151 & 0.32 & 2.23 & 0.00 & 0.00 \\
$128-256$ & 674 & 2,365 & 64,982 & 45,369 & 3.35 & 3.78 & 20.50 & 8.21 \\
$257-512$ & 2,154 & 5,162 & 88,035 & 121,868 & 10.70 & 8.26 & 27.77 & 22.05 \\
$513-1,024$ & 3,897 & 10,476 & 54,326 & 131,931 & 19.35 & 16.76 & 17.13 & 23.87 \\
$1,025-2,048$ & 4,593 & 13,572 & 39,500 & 118,958 & 22.81 & 21.72 & 12.46 & 21.52 \\
$2,049-4,096$ & 4,566 & 13,990 & 35,656 & 88,667 & 22.67 & 22.39 & 11.25 & 16.04 \\
$4,097-8,192$ & 3,263 & 11,530 & 27,919 & 38,658 & 16.20 & 18.45 & 8.81 & 6.99 \\
$8,193-16,384$ & 850 & 3,547 & 6,450 & 6,481 & 4.22 & 5.68 & 2.03 & 1.17 \\
$>16,385$ & 76 & 464 & 193 & 650 & 0.38 & 0.74 & 0.06 & 0.12 \\
total & 20,317 & 62,497 & 317,062 & 552,733 & 100 & 100 & 100 & 100 \\
\hline
\end{tabular}

Table S3. Unique protein length distribution of different modeling programs

\begin{tabular}{c|cccc|cccc}
\hline \multirow{2}{*}{$\begin{array}{c}\text { size range } \\
(\text { aa })\end{array}$} & \multicolumn{4}{|c|}{ frequency } & \multicolumn{4}{c}{ percentage } \\
\cline { 2 - 9 } & MAKER2 & Cufflinks & Trinity & Oases & MAKER2 & Cufflinks & Trinity & Oases \\
\hline$<64$ & 580 & 869 & 1,740 & 4,737 & 2.60 & 2.33 & 3.02 & 3.63 \\
$64-128$ & 4,825 & 6,175 & 13,293 & 39,111 & 21.63 & 16.55 & 23.08 & 29.98 \\
$129-256$ & 5,201 & 7,026 & 11,437 & 35,350 & 23.31 & 18.83 & 19.86 & 27.09 \\
$257-512$ & 6,460 & 11,859 & 16,816 & 32,388 & 28.96 & 31.78 & 29.20 & 24.82 \\
$513-1,024$ & 3,771 & 7,820 & 10,255 & 14,580 & 16.90 & 20.96 & 17.81 & 11.17 \\
$1,025-2,048$ & 1,212 & 2,711 & 3,362 & 3,729 & 5.43 & 7.27 & 5.84 & 2.86 \\
$2,049-4,096$ & 232 & 626 & 592 & 515 & 1.04 & 1.68 & 1.03 & 0.39 \\
$4,097-8,192$ & 25 & 159 & 83 & 52 & 0.11 & 0.43 & 0.14 & 0.04 \\
$8,193-16,384$ & 3 & 61 & 15 & 12 & 0.01 & 0.14 & 0.03 & 0.00 \\
$16,385-32,768$ & 1 & 10 & 0 & 0 & 0.00 & 0.03 & 0.00 & 0.00 \\
\hline total & 22,310 & 37,316 & 57,593 & 130,474 & 100 & 100 & 100 & 100 \\
\hline
\end{tabular}

Table S4. Length distribution of the Cufflinks 3.0 proteins with $\mathrm{P} / \mathrm{N} / \mathrm{O}$ or $\mathrm{B} / \mathrm{W}$ matches

\begin{tabular}{c|cc|cc}
\hline \multirow{2}{*}{ length } & \multicolumn{2}{|c|}{ count } & \multicolumn{2}{c}{ percentage } \\
\cline { 2 - 5 } & $\mathrm{B} / \mathrm{W}$ & $\mathrm{P} / \mathrm{N} / \mathrm{O}$ & $\mathrm{B} / \mathrm{W}$ & $\mathrm{P} / \mathrm{N} / \mathrm{O}$ \\
\hline$<64$ & 293 & 94 & 15.07 & 1.33 \\
$64-128$ & 1,042 & 802 & 53.60 & 11.31 \\
$129-256$ & 258 & 1,266 & 13.27 & 17.85 \\
$257-512$ & 296 & 2,796 & 15.23 & 39.41 \\
$513-1024$ & 40 & 1,708 & 2.06 & 24.08 \\
$1,025-2,048$ & 15 & 382 & 0.77 & 5.38 \\
$2,049-4,096$ & 0 & 40 & 0 & 0.56 \\
$4,097-8,192$ & 0 & 6 & 0 & 0.08 \\
\hline
\end{tabular}


Table S5. Length distribution of Cufflinks 3.0 proteins with different numbers of hits in UniProt

\begin{tabular}{c|cccccc}
\hline \multirow{2}{*}{ length } & \multicolumn{7}{|c}{ match number } \\
\cline { 2 - 7 } & 0 & 1 & 2 & 3 & 4 & 5 \\
\hline$<64$ & 524 & 53 & 44 & 28 & 21 & 199 \\
$64-128$ & 2,625 & 448 & 473 & 309 & 326 & 1,994 \\
$129-256$ & 1,174 & 636 & 838 & 523 & 661 & 3,194 \\
$257-512$ & 1,590 & 1,092 & 1,259 & 806 & 1,234 & 5,878 \\
$513-1,024$ & 827 & 945 & 1,061 & 561 & 874 & 3,552 \\
$1,025-2,048$ & 223 & 334 & 506 & 238 & 270 & 1,140 \\
$2,049-4,096$ & 36 & 106 & 89 & 63 & 97 & 235 \\
$>4,096$ & 4 & 101 & 33 & 37 & 26 & 29 \\
\hline total & 7,003 & 3,715 & 4,303 & 2,565 & 3,509 & 16,221 \\
\hline
\end{tabular}




\section{Graphic Abstract}

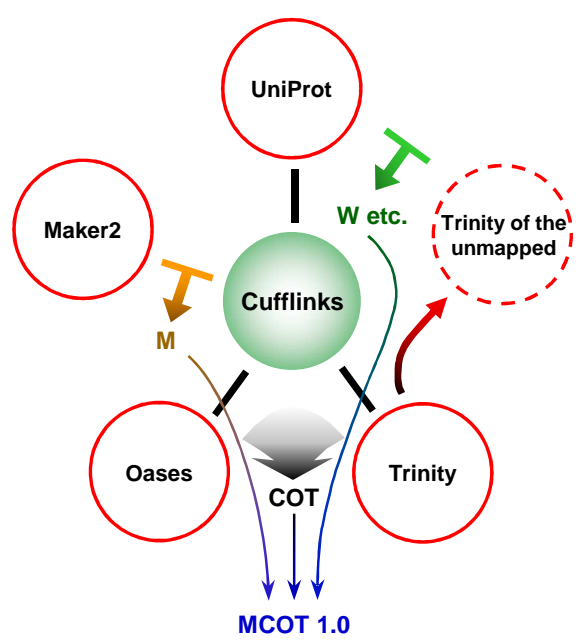

Fig. 1

A

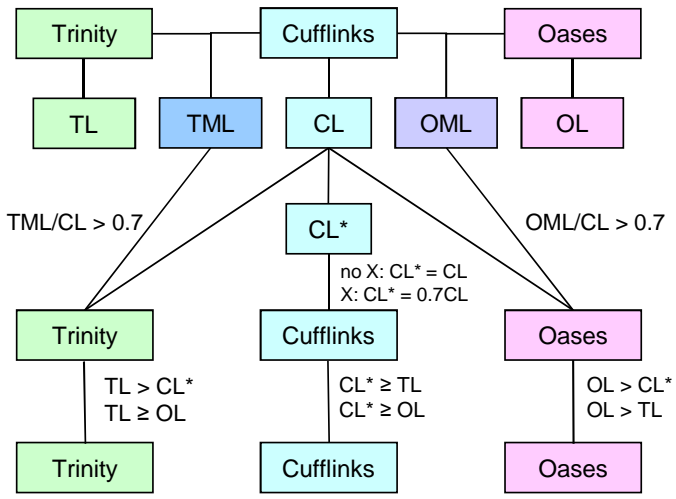

Selection 1:
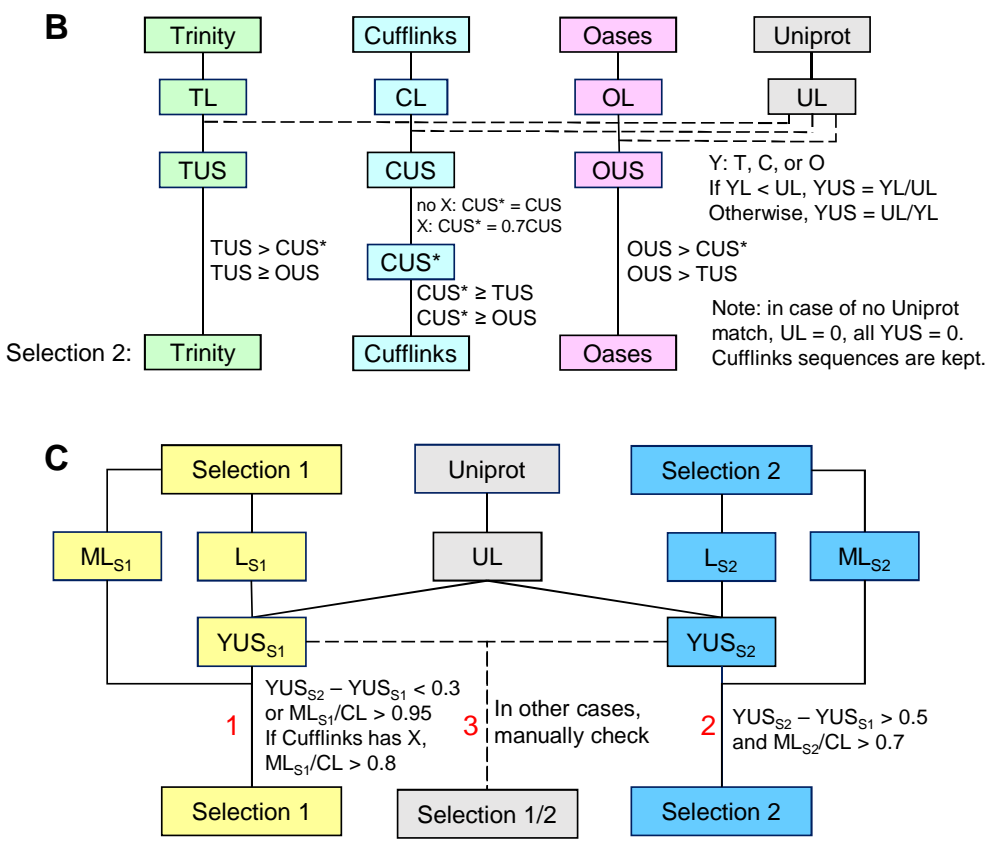
Fig. 2

A
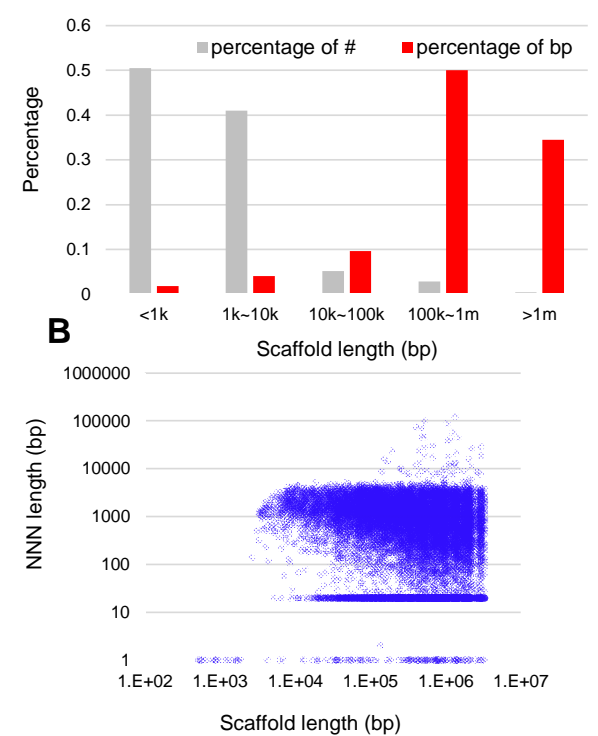

Fig. 3

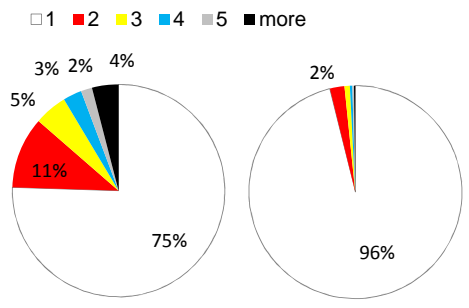

Fig. 4

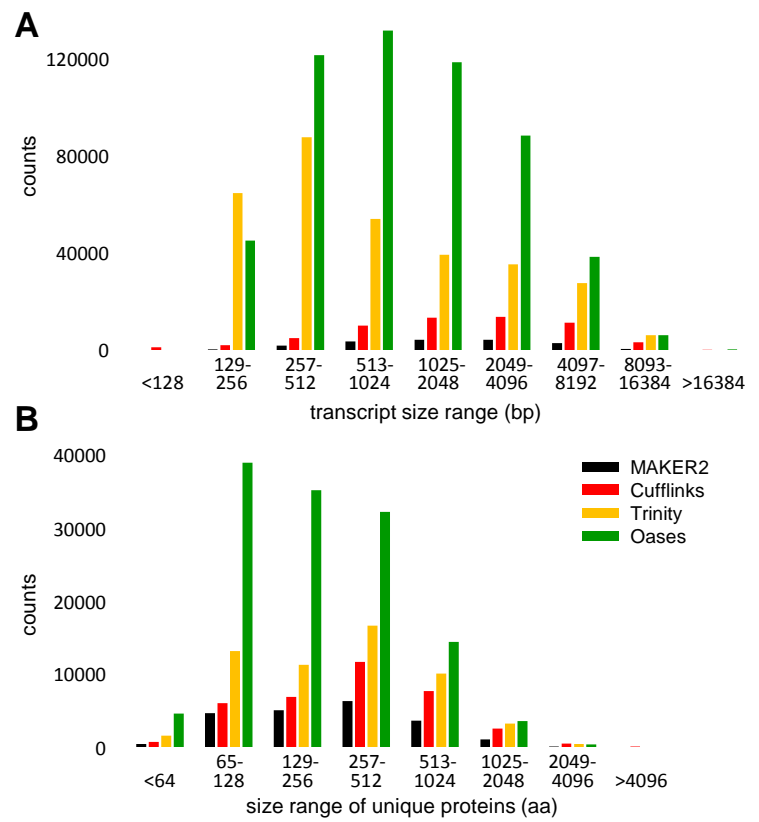

Fig. 5
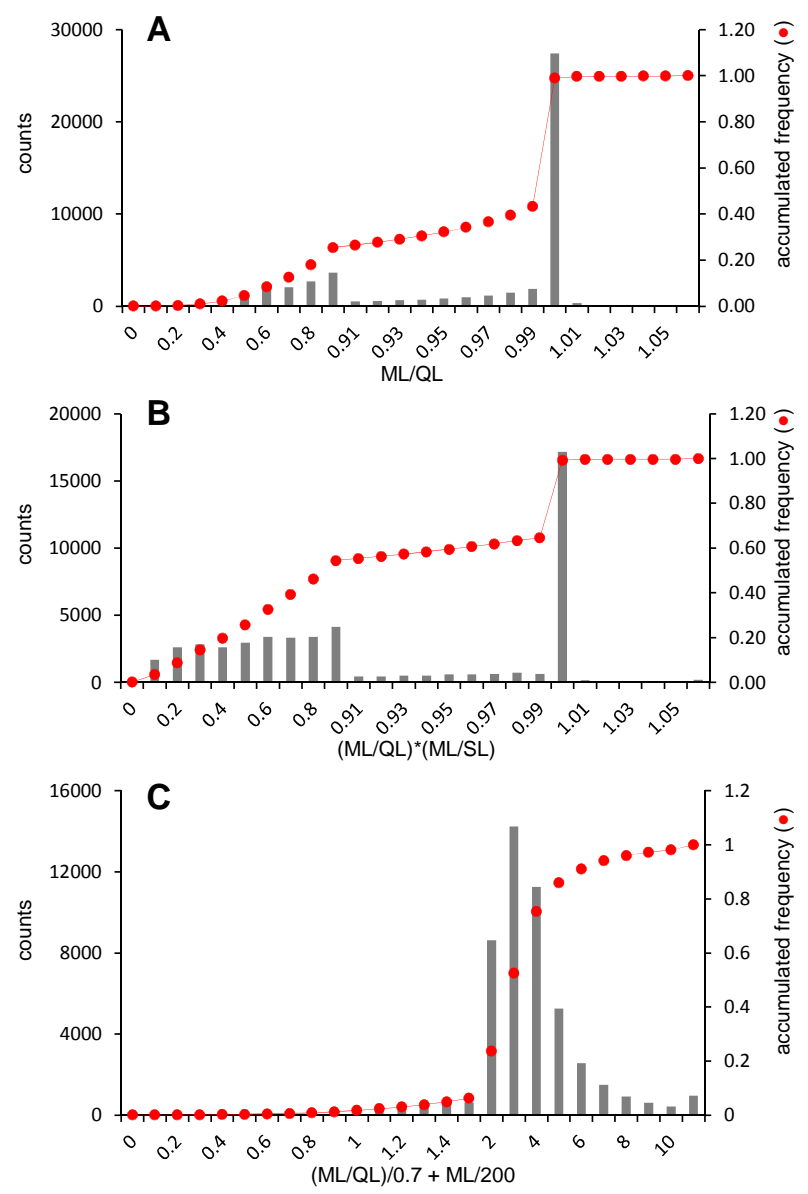

Fig. 6

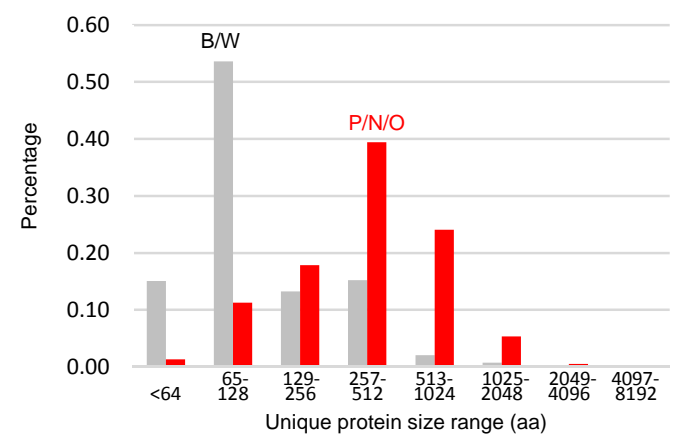

Fig. 7

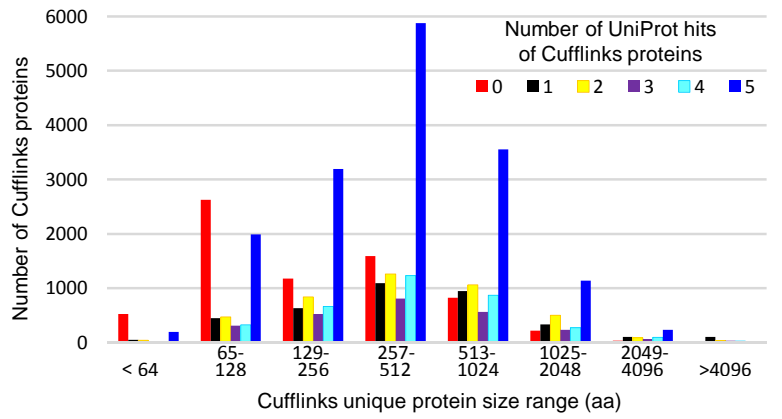


Fig. 8

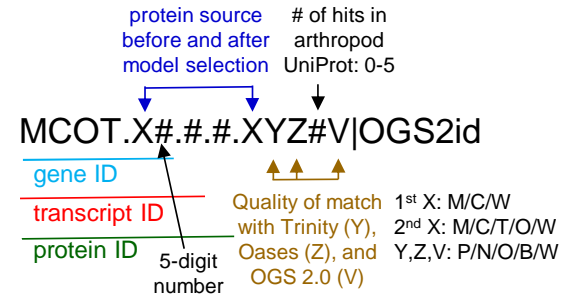

Fig. 9

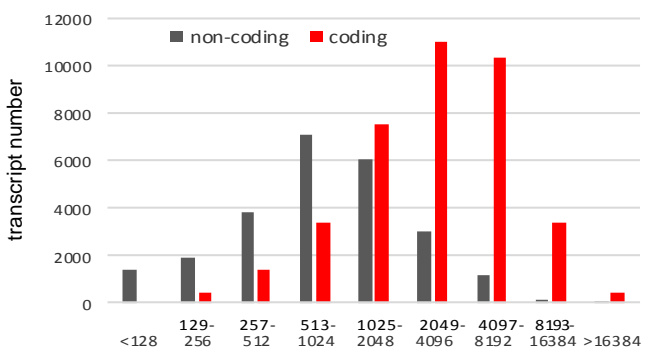

